\title{
ASSOCIAÇÃO ENTRE O INDÍCE DE MASSA CORPORAL, JORNADA DE TRABALHO E NÍVEL DE ATIVIDADE FÍSICA DOS SERVIDORES DO HEMOCENTRO DO HOSPITAL UNIVERSITÁRIO DE MARINGÁ
}

\author{
Pâmela Norraila da Silva ${ }^{1}$ \\ Majorie Catherine Capdeboscq ${ }^{2}$ \\ Ana Carolina Belther Santos \\ Ana Silvia Degasperi Ieker ${ }^{4}$ \\ Wilson Rinaldi ${ }^{5}$
}

SILVA, P. N. da; CAPDEBOSCQ, M. C.; SANTOS, A. C. B.; IEKER, A. S. D.; RINALDI, W. Associação entre o indíce de massa corporal, jornada de trabalho e nível de atividade física dos servidores do hemocentro do hospital universitário de maringá. Arq. Cienc. Saúde UNIPAR, Umuarama, v. 20, n. 3, p, 165-170, set./dez. 2016.

\begin{abstract}
RESUMO: O profissional de saúde está sujeito a diversos comportamentos de risco que são determinantes para o desenvolvimento de doenças agudas e crônicas no decorrer da vida, sendo que o estilo de vida adotado influencia diretamente nesse contexto. O objetivo deste estudo foi associar o índice de massa corporal, a jornada de trabalho e nível de atividade física dos servidores do Hemocentro do Hospital Universitário de Maringá. Esta pesquisa caracteriza-se do tipo transversal, contando com a participação de 32 servidores. Os dados foram analisados por estatística descritiva de frequência absoluta e percentual, e o Teste Qui-Quadrado para associação das variáveis por meio do pacote estatístico SPSS, adotando um $\mathrm{p}<0,05$. Nos resultados encontrados, $56,2 \%$ dos servidores foram classificados como não ativos, e $28,1 \%$ foram classificados com sobrepeso. Diante deste estudo pode-se verificar a importância de programas de intervenção no ambiente de trabalho, como ginástica laboral durante a jornada de trabalho, ou atividades físicas após ou antes a jornada laboral, para prevenção de doenças e promoção da saúde.
\end{abstract}

PALAVRAS-CHAVE: Atividade física. Jornada de trabalho. Saúde.

\section{ASSOCIATION AMONG BODY MASS INDEX, WORK DAY AND LEVEL OF PHYSICAL ACTIVITY OF WORKERS OF THE BLOOD CENTER AT THE UNIVERSITY HOSPITAL OF MARINGÁ}

\begin{abstract}
Health professionals are subject to various risk behaviors that are determinants to the development of acute and chronic diseases later in life, with direct influence of the adopted lifestyle. The purpose of this study was to associate the body mass index, the working shift and the level of physical activity of health professionals working at the Blood Center of the University Hospital of Maringá. This is a cross-sectional study with 32 participants. Data were analyzed using descriptive statistics of absolute and percentage frequency, as well as the Chi-Square test for association of variables using the SPSS statistical package, adopting $\mathrm{p}<0.05$. The results showed that $56.2 \%$ of workers were classified as sedentary, while $28.1 \%$ were classified as overweight. Therefore, the importance of intervention programs in the workplace is emphasized, such as occupational gymnastics during the working day, or physical activities after or before the work shift, in order to prevent diseases and promote the health of the workers.
\end{abstract}

KEYWORDS: Health. Physical activity. Working hours.

\section{Introdução}

$\mathrm{Na}$ década de 30, havia prevalência de doenças infectocontagiosas devido às condições ambientais em que as pessoas se encontravam. Porém, com advento das altas tecnologias, ocorreu o surgimento de algumas vacinas como forma de diminuir a prevalência de doenças infectocontagiosas (MALTA et al., 2006). Com as industrializações e urbanizações, um novo estilo de vida foi adotado e, consequentemente, houve um aumento no número de pessoas com problemas de saúde. Esse processo de industrialização fez com que acontecesse uma configuração na transição nutricional, no qual reduziu o número de casos com desnutrição e aumentou a frequência do excesso de peso (RAMOS; DUMITH; CÉSAR, 2015).

Segundo Kac; Velásquez-Meléndez (2003), a transição nutricional configura como uma "uma mudança na mag- nitude e no risco atribuível de agravos associados ao padrão de determinação de doenças atribuídas ao atraso e a modernidade, sendo em geral, uma passagem da desnutrição para a obesidade" (p. S4). Ainda, houve um aumento no consumo de alimentos com alto teor energético, e quando associados à diminuição de atividade física, convergiram no aumento de pessoas com doenças crônicas não transmissíveis (DCNT) (MARIATH et al., 2007; BIELEMANNL et al., 2015). O aumento da prevalência das DCNT se tornou uma epidemia mundial, gerando uma grande preocupação na área de saúde pública. Entre as DCNT, destacam-se a prevalência de obesidade, câncer, hipertensão arterial, colesterol alterado, que aumentaram exponencialmente (SIQUEIRA, 2009; SILVA et al., 2015). Segundo Santos; Duarte (2009), vários fatores de riscos devem ser considerados como, por exemplo, idade, sexo, baixa escolaridade, desigualdades sociais e diferença no acesso aos bens e informação, como fatores que estão li-

DOI: https://doi.org/10.25110/arqsaude.v20i3.2016.5668

${ }^{1}$ Mestranda em Educação Física na linha de fatores psicossocias e motores do desempenho humano pelo Programa de Pós- Graduação associado UEM/UEL. Endereço para correspondência: Rua Yruama, 1536- Bairro Iguatemi - CEP: 87103-232 - Maringá - Paraná - Email: pamelanorraila@gmail.com

${ }^{2}$ Mestre em Educação Física na linha Atividade Física e Saúde pelo Programa de Pós-Graduação associado UEM/UEL. Gerente de Promoção e Prevenção em Saúde da Secretaria Municipal de Saúde de Maringá/PR. Email: majoriecc@hotmail.com

${ }^{3}$ Graduanda em Educação Física pela Universidade Estadual de Maringá. Email: ana_belther_1993@yahoo.com.br

${ }^{4}$ Mestranda em Educação Física na linha Atividade Física e Saúde pelo Programa de Pós-Graduação associado UEM/UEL. Email: anasilviaieker@hotmail. com

${ }^{5}$ Doutorado em Ciências Biológicas pela Universidade Estadual de Maringá, professor do departamento de Educação Física. Endereço para correspondência: Avenida Colombo, Zona 7, 87020-000 - Maringá, PR - Brasil Telefone: (44) 3261-4315. Email: wilsonrinaldi@hotmail.com 
gados ao aparecimento de doenças crônicas nos indivíduos (ROCHA-BRISCHILIARI et al., 2014).

Ademais, as exigências do trabalho podem ser um dos fatores que acarretam doenças crônicas, já que as condições de trabalho apresentado aos profissionais da saúde são inadequadas e de grande preocupação (LIMA JÚNIOR; ALCHIERI; MAIA, 2009). É perceptível que há uma preocupação com estes indivíduos, devido estes permanecerem em um ambiente voltado para "cura" de pessoas, e acaba se tornando um ambiente propício para o adoecimento dos que ali trabalham, pois oferece riscos de acidente e à doenças (ELIAS; NAVARRO, 2006; KARINO et al., 2015).

Os profissionais que atuam nos âmbitos hospitalares sofrem sobrecargas de trabalho que afetam o físico e o emocional destes sujeitos, podendo levar a um sofrimento psíquico, como crises depressivas, de ansiedade e de estresse (FRONZA; TEIXEIRA, 2010; SILVA et al., 2006; SILVA; ROTENBERG; FISCHER, 2011; SANTANA et al., 2013; KARINO et al., 2015). A carga horária de serviço também pode interferir negativamente na vida do trabalhador, fazendo com que os trabalhadores diminuam seu tempo de sono, desenvolvimento de doenças, como estresse e obesidade, além de não encontrarem tempo para o lazer ou atividade física (PAFARO; MARTINO, 2004; MORENO; FISCHER; ROTENBERG, 2003; SILVA et al., 2010; FREIRE et al., 2015). É importante ressaltar que a ausência de atividade física, a prevalência de doenças crônicas, aumento do quadro de morbimortalidade estão relacionados às modificações e organizações do mercado trabalho (CAVALCANTE et al., 2006; FREIRE et al., 2015), que interferem negativamente na qualidade de vida do trabalhador.

$\mathrm{Na}$ literatura há estudos voltados para os profissionais da saúde, mas são escassos os estudos na região de Maringá e que investiguem as condições de saúde e a prática de atividade física dos profissionais da saúde. Desta forma, o objetivo deste trabalho é associar o índice de massa corporal, jornada de trabalho e nível de atividade física dos servidores do setor Hemocentro do Hospital Universitário de Maringá.

\section{Materiais e Métodos}

De acordo com Thomas; Nelson; Silverman (2007), esta pesquisa do tipo transversal, possui como característica central, a observação, o registro, a análise, a descrição, correlação de fatos/fenômenos sem manipular, buscando explicação para a frequência em que ocorrem os fatos e a sua relação deste com outros fatores. O estudo em questão está vinculado ao projeto pesquisa Análise do nível de atividade física, hábitos alimentares e estilo de vida dos servidores da Universidade Estadual de Maringá, aprovado pelo Comitê Permanente de Ética em Pesquisa (COPEP) da Universidade Estadual de Maringá sob número de processo 517/2009. Foram entregues Termos de Consentimento Livre e Esclarecido (TCLE) para todos os funcionários do setor Hemocentro do Hospital Universitário de Maringá (HUM) no Paraná, sendo 68 servidores tanto efetivos, como regime provisório, porém somente 32 funcionários aceitaram participar desta pesquisa voluntariamente e entregaram o TCLE assinado corretamente no dia da coleta de dados.

A coleta dos dados foi realizada entre os meses de dezembro de 2012 a março de 2013, por meio de aplicação de questionários e avaliação física para obter os dados antropométricos dos funcionários do hemocentro do Hospital Universitário de Maringá - HUM. O local para aplicação dos questionários foi em um anfiteatro do hemocentro, sendo que cada sujeito recebeu um questionário para auto resposta. Um pesquisador estava presente na aplicação da anamnese para, caso necessário, sanar dúvidas que surgissem no decorrer do preenchimento.

Para as avaliações físicas, os acadêmicos que participam do projeto de pesquisa e extensão + UEM - Promoção da Saúde, vinculados ao Pró-ação, coletaram os dados dos indivíduos do estudo. Estes acadêmicos receberam um treinamento específico para a realização da coleta de dados. Dividiu-se em estações para mensurar as variáveis antropométricas, no qual um mesmo acadêmico ficou responsável por aferir uma variável de todos os sujeitos do estudo, para diminuir o grau de erro. Os sujeitos passavam em cada estação, tendo seus dados coletados e transcritos em suas fichas individuais. A coleta de dados dos servidores só aconteceu depois da assinatura dos TCLE pelos servidores participantes.

Os participantes tiveram a estatura $(\mathrm{cm})$ aferida com estadiômetro inglês, tipo Leicesters, acoplado a uma base que permite medida de altura em campo, fabricado pela Child Growth Foundation, a circunferência da cintura $(\mathrm{cm})$ aferida por meio de uma trena antropométrica (CESCORF®) na altura do umbigo. O peso corporal foi medido em balança da marca Filizola, com capacidade máxima de $150 \mathrm{~kg}$. O cálculo do IMC foi realizado por meio da razão entre o peso $(\mathrm{Kg})$ pela estatura $(\mathrm{cm})$ ao quadrado. Para classificação do IMC, foram considerados: baixo peso (IMC $<18,5$ ), normal $(18,5 £$ IMC $<24,9)$, sobrepeso $(25 £$ IMC $<29,9)$ e obesidade (IMC >30) (AFONSO; SICHIERI, 2002).

Com relação aos instrumentos de medida utilizados, foi aplicado um questionário sobre as condições sociodemográficas e de saúde, para levantamento das informações necessárias para análise. As questões referiam-se às informações pessoais, histórico de saúde pessoal e familiar, hábitos de vida, hábitos de atividade física e dados demográficos. Outro questionário aplicado para avaliar o nível de atividade física dos sujeitos foi o International Physical Activity Questionnaire (IPAQ), versão 8, no qual são questões relacionadas à frequência (dias por semana), a duração (tempo por dia) e o tipo de atividades físicas, que podem ser leves, moderadas e vigorosas.

O instrumento IPAQ classifica os sujeitos em "inativo", "irregularmente ativo", "ativo" e "muito ativo", seguindo os critérios de frequência, duração e tipo de atividade física. Neste estudo os servidores foram classificados seguindo esta metodologia e após as categorias "inativo" e "irregularmente ativo" foram agrupado, assim como "ativo" e "muito ativo" para análises estatísticas dos dados.

$\mathrm{Na}$ análise dos dados, estes foram expressos por meio de estatística de frequência absoluta e relativa. Para associação das variáveis foi utilizado o Teste Qui- Quadrado, por meio do pacote estatístico SPSS, adotando um $\mathrm{p}<0,05$.

\section{Resultados}

Nos resultados obtidos é constatado que $71,9 \%$ dos sujeitos são do sexo feminino e também que $75 \%$ possuem 
ensino superior. A maioria dos sujeitos do estudo é casado $(65,5 \%)$ e trabalham de seis a oito horas por dia $(75 \%)$. Quando questionado sobre a atividade física, 59,4\% dos sujeitos afirmaram praticar qualquer atividade física, entretanto na classificação do instrumento o questionário IPAQ, 56,2\% dos servidores participantes do estudo foi classificado como não ativos. Para aprofundar a análise, foi investigado o índice de massa corporal dos servidores, e foi verificado que as mulheres apresentaram uma média de peso de $69,5 \mathrm{~kg}$, já os homens apresentaram uma média de $84,4 \mathrm{~kg}$. Em relação ao IMC dos servidores $37,5 \%$ foi classificado em eutrófico e $28,1 \%$ com sobrepeso, sendo $43,5 \%$ das mulheres considerada normal dentro dos números classificados no estado nutricional e os servidores do sexo masculino prevaleceu a classificação sobrepeso $(44,4 \%)$.

Como foi possível calcular o IMC, procurou-se verificar se a prática/ausência de atividade física (AF) influencia no índice de massa corporal, ou seja, associar o IMC e AF. Conforme a tabela 1, foi possível verificar que indivíduos que não praticam qualquer atividade física $46,2 \%$ são classificados em baixo peso/ normal e $63,2 \%$ sobrepesados/ obesos. Também foi notável que $53,8 \%$ da população do estudo praticam alguma atividade física e mantém o peso corporal dentro dos padrões eutróficos, enquanto que $36,8 \%$ se exercitam, mas são sobrepesados ou obesos.

Tabela 1: Associação do índice de massa corporal e o nível de atividade física dos servidores do setor hemocentro - HUM.

\begin{tabular}{c|cccccc}
\hline \multicolumn{2}{c}{} & \multicolumn{7}{c}{ IPAQ } & & & \\
& & Ativos & & Não ativos & & Total \\
\cline { 3 - 8 } & & $\mathrm{N}$ & $(\%)$ & $\mathrm{N}$ & $(\%)$ & \\
\hline \multirow{2}{*}{ IMC } & Baixo peso/ Normal & 5,7 & 53,8 & 7,3 & 46,2 & $13(100,0)$ \\
& Sobrepeso e obesidade & 8,3 & 36,8 & 10,7 & 63,2 & $19(100,0)$ \\
\hline
\end{tabular}

Para completar a análise, associou-se a jornada de trabalho com o índice de massa corporal e verificou-se que a maioria dos sujeitos trabalha de seis a oito horas, entretanto $46,7 \%$ desses indivíduos é sobrepesado/obeso, enquanto que apenas $26,7 \%$ foi classificado com o índice de massa corporal baixo peso/ normal. Com relação aos indivíduos que trabalham mais de oito horas, houve uma equivalência nos resultados, em que $13,3 \%$ foi considerado baixo peso/ nor- mal e 13,3\% sobrepeso/obesidade. Associando a jornada de trabalho ao nível de atividade, é observável que 40,6\% da população deste estudo trabalham de seis a oito horas por dia e é não ativo, enquanto que $34,4 \%$ trabalham de seis a oito horas por dia, porém é classificado como ativo. Já os servidores que trabalham mais de oito horas, apenas $9,4 \%$ foram considerados como ativo e $15,6 \%$ não ativo.

Tabela 2: Associação entre a jornada de trabalho, o índice de massa corporal e o nível de atividade física dos servidores do hemocentro do HUM.

\begin{tabular}{|c|c|c|c|c|c|c|}
\hline & & \multicolumn{4}{|c|}{ Jornada de trabalho } & \multirow{3}{*}{ Total } \\
\hline & & \multicolumn{2}{|c|}{6 a 8 horas } & \multicolumn{2}{|c|}{ Mais de 8 horas } & \\
\hline & & $\mathrm{N}$ & $(\%)$ & $\mathrm{N}$ & $(\%)$ & \\
\hline \multirow[t]{2}{*}{ IMC } & Baixo Peso/ Normal & 8 & 26,7 & 4 & 13,3 & $12(40,0)$ \\
\hline & Sobrepeso/ Obesidade & 14 & 46,7 & 4 & 13,3 & $18(60,0)$ \\
\hline \multirow[t]{2}{*}{ IPAQ } & Ativo & 11 & 34,4 & 3 & 9,4 & $14(43,8)$ \\
\hline & Não Ativo & 13 & 40,6 & 5 & 15,6 & $18(56,2)$ \\
\hline
\end{tabular}

\section{Discussão}

O objetivo deste trabalho é associar o índice de massa corporal, jornada de trabalho e nível de atividade física dos servidores do setor Hemocentro do Hospital Universitário de Maringá. Desta forma, foi encontrado que a maioria dos sujeitos do estudo classificado em sobrepeso/ obeso, $63,2 \%$ é inativo e $46,7 \%$ trabalha de 6 a 8 horas por dia . Autores apontam que numa jornada de trabalho árdua, os efeitos negativos podem ser imediatos, como sintomas de fadiga, má alimentação, estresse, dores corporais (SILVA; ROTENBERG; FISCHER, 2011), e muitos indivíduos não praticam qualquer atividade física e são considerados, dentro das classificações de Afonso; Sichieri (2002), sobrepesados ou obesos.

Em estudo realizado por Matos; Proença (2003) em uma Unidade de Alimentação e Nutrição, surgiu uma preocupação com a saúde dos trabalhadores relacionada às condições de trabalho e desempenho na produtividade. Foi verificado nessa empresa, que os servidores revelaram um alto índice de sobrepeso e suposto que este aumento corporal ocorreu após o início das atividades nessa unidade de trabalho, além de uma mudança nos hábitos alimentares. Siqueira (2009) alega que há a falta de aconselhamento sobre a prática de exercícios físicos e seus benefícios, principalmente entre os próprios profissionais da saúde que trabalham em hospitais. O autor ainda aponta que no Brasil ainda existe a necessidade de aprimoramento de conhecimentos para a atividade física, o cuidado com a saúde, a prevenção e como ajuda no tratamento de enfermidades, como a hipertensão.

Com a investigação do item atividade física, o questionário IPAQ apontou que $56,2 \%$ de sujeitos como não ativos. Segundo Mantovani (s/d) para que as pessoas pratiquem regularmente algum tipo de atividade física é necessário que elas tenham conhecimentos suficientes sobre os benefícios advindo desta prática. Entretanto, Arraias; Nunes; Oliveira 
(2013) analisaram a percepção que os profissionais da saúde possuem sobre a prática de atividade física, e encontraram evidências de que todos possuem conhecimento da importância da prática de atividade física, principalmente os profissionais que não praticavam qualquer atividade física. As percepções acerca do assunto foram desde o conhecimento sobre os benefícios da prática de atividade física na qualidade de vida até prevenção de doenças associadas ao sedentarismo.

É certo que a população conhece os benefícios de tal prática, entretanto são diversos os motivos que levam os sujeitos a não praticarem exercícios físicos. Em um estudo realizado com policiais por Jesus; Jesus (2012), os sujeitos relataram não praticar atividade física devido a jornada de trabalho $(36,7 \%)$, também por compromissos familiares $(39,2 \%)$, falta de equipamento $(30,4 \%)$, ambiente inseguro $(26,9 \%)$, falta de companhia $(25,6 \%)$, tarefas domésticas $(20,6 \%)$ e falta de recursos financeiros $(20,3 \%)$. Santics (2008) coloca que a inatividade física reflete nas condições de trabalho, por isso que as empresas começaram a se preocupar com o que a empresa oferece, avançando nas questões tecnológicas da empresa. Entretanto o autor completa que na saúde do trabalhador, essas tecnologias contribuem apenas para adoção de um estilo de vida inativo e com alimentação hipercalórica.

Costa (s/d) aponta que com o advento da industrialização e uma alta jornada de trabalho é perceptível o decréscimo do nível de atividade física no trabalho, o que resulta na proliferação de doenças advindas do trabalho. O trabalho em hospitais, segundo Pafaro; Martino (2004), contém riscos para a saúde principalmente quando o trabalho é realizado em período noturno e, Silva; Queiroz (2011) completa que o trabalho noturno causa uma redução no período de sono e então está associado ao aumento de acidentes no trabalho. Silveira; Gattelli; Moura (2012) afirmam que como os profissionais da saúde, dão assistência necessária aos pacientes, é exigida a manipulação de equipamentos pesados, preparação de medicamentos e a frequente utilização de instrumentos perfurantes e cortantes.

Entretanto a busca pela produtividade e a alta jornada de trabalho desenvolvem consequências principais como fadiga, cansaço físico e metal, estresse, fadiga muscular na parte solicitada durante a jornada (FARIA JÚNIOR, 1990). Silva; Queiroz (2011) abrandam que a maior parte das pesquisas enfatizam o estresse no trabalho nos profissionais da saúde, entretanto com os duplos cargos pela modalidade de trabalho por divisão de plantões, as pessoas tendem a diminuir o tempo das atividades física e de lazer (FREIRE et al., 2015). Podemos supor que, esse elevado número de sujeitos do estudo que não praticam atividade física classificados pelo instrumento IPAQ possuam o motivo descrito por Faria Júnior (1990) e Silva; Queiroz (2011) ou devido a atividades domésticas.

Lara; Soares; Juvêncio (2009) confirmam a ideia de que no meio empresarial está aumentando o interesse pelas empresas que perceberam que a produtividade está relacionada a qualidade de vida dos trabalhadores. Os autores compartilham a ideia de que os empresários tentam solucionar este problema de doenças relacionada ao trabalho com a implantação de programas de exercícios esportivos para os empregados, inserindo a ginástica laboral no ambiente de trabalho, pois é uma ferramenta de curta duração, na hora do expediente, que não leva o trabalhador ao cansaço compensando as estruturas musculares envolvidas na tarefa (LARA; SOARES; JUVÊNCIO, 2009), ou ainda são construídas academias nas empresa (SANCTICS et al., 2008).

São encontrados na literatura estudos em grande escala sobre a prática de atividade física, entretanto são poucos os que abordam sobre a prática de atividade física dos profissionais da saúde. Conceição et al. (2006) encontrou em seu estudo com funcionários da Universidade de Brasília no ano de 2002, uma alta prevalência de sedentarismo (48,4\%) nos indivíduos que relatavam que não praticavam nenhum tipo de atividade física durante a semana. Bernardo et al. (2013) estudou um grupo de funcionários de uma Universidade Pública usando as categorias do inventário de Baecke, e foi encontrado que a maioria dos sujeitos estudados foram considerados como sedentário nas categorias de atividade física ocupacional e atividade física total, e quando foram analisados, segundo o inventário nas categorias de atividade física de tempo livre e atividade física de lazer, ainda assim foram classificados como sedentários.

É fato que uma alta prevalência de sedentarismo influencia no peso corporal dos sujeitos e neste estudo, foram constatados que a maioria das mulheres está com o estado nutricional classificadas como normal $(43,5 \%)$, enquanto que os homens estão com sobrepeso $(44,4 \%)$, sendo que de maneira geral, o estado nutricional constatado foi normal (37,5\%). Coutinho e Cols apud Acuña e Cruz (2004), concluiram em seus estudos que pessoas com baixo peso apresentaram $15,4 \%$ para homens e $16,5 \%$ para mulheres, sendo encontrada a maior prevalência de baixo peso no Nordeste $(20 \%)$ e menor na região Sul $(10 \%)$. A outra parcela do estudo apresentou $24,6 \%$ de sobrepeso e $8,3 \%$ de obesidade, em que as mulheres foram as mais afetadas (38\%). Na região norte o excesso de peso se portou como um grande problema e na região Sul em $34 \%$ dos homens e $43 \%$ das mulheres.

Rezende et al. (2006) estudou servidores de uma Universidade e encontrou que $42,5 \%$ das mulheres estavam com sobrepeso, 24,5\% classificadas com obesidade, enquanto que os homens, $40 \%$ com sobrepeso e $15,2 \%$ com obesidade. Os autores ainda completam que o sedentarismo é um dos grandes fatores de risco para a obesidade no estudo. Nesse sentido Neta; Fernandes Filho; Cortez (2016) estudaram o perfil dos policiais e encontrou que a maioria era inativa fisicamente, e como consequência, o estado de sobrepeso estava aumentando nesta categoria. Conclui-se que a inatividade física acarreta diversos prejuízos a saúde.

Algumas limitações do estudo são importantes ressaltar, como: o estudo foi realizado com servidores do Hospital Universitário de Maringá, no seu ambiente de trabalho, nos dias de coleta muitos deles não estavam com roupas adequadas para a coleta; a coleta tinha de ser rápida, para que o servidor voltasse as suas atividades; e os servidores tiveram a total liberdade para não participar do estudo.

\section{Conclusão}

Este trabalho buscou associar o índice de massa corporal, a jornada de trabalho e nível de atividade física dos servidores do Hemocentro do Hospital Universitário de Maringá, no qual a maioria dos sujeitos do estudo trabalham de seis a oito horas por dia e estão sobrepesados/obesos e 
inativos. O estudo serviu para atentar os servidores sobre as condições de saúde impostas, prevenir de algumas doenças prováveis de acontecer no decorrer da vida laboral e promover a saúde para estes servidores do Hemocentro .

Diante deste estudo podemos verificar também a importância de programas de intervenção no ambiente de trabalho, como ginástica laboral durante a jornada de trabalho, ou atividades físicas após o trabalho, pois se acredita que a jornada de trabalho seja um fator importante para o sobrepeso presente no estudo no sexo masculino e o baixo índice de sujeitos considerados ativos. Acredita-se também na importância de equipes multiprofissionais em qualquer ambiente de trabalho (médicos, enfermeiros, nutricionistas, assistentes sociais, psicólogos, professores de educação física e funcionários administrativos) para que se tenha um cuidado totalitário da vida desse trabalhador.

Esse trabalho contribuiu qualitativamente para a literatura, pois são escassos os estudos que abordam a saúde do trabalhador dentro do Hospital Universitário de Maringá, no setor hemocentro. Entretanto, há uma carência de programas institucionais dentro das universidades que acompanham a vida do trabalhador, por um longo período de tempo, que consequentemente verifica melhoras na saúde e na qualidade de vida do trabalhador. Sugere-se outros estudos que investiguem os fatores influenciadores na prática de atividade física e no índice de massa corporal, pois há a necessidade de desvendar a contradição encontrada no questionário aplicado com os servidores. É certo que, mesmo o questionário classificando os servidores como não ativos, estes não buscam somente uma melhora física, mas também um bem estar físico, psíquico e social.

\section{Referencias}

ACUÑA, K; CRUZ, T. Avaliação do Estado Nutricional de Adultos e Idosos e Situação Nutricional da População Brasileira. Revista Arquivos Brasileiros de Endocrinologia \& Metabologia, 48(3): 345- 361, Jun 2004.

AFONSO, F. M.; SICHIERI, R. Associação do índice de massa corporal e da relação cintura/quadril. Revista Brasileira de Epidemioliologia, 5(2), 153-163, 2002.

ARRAIS, P. S. D.; NUNES, R. J.; OLIVEIRA, Y. S. Percepção dos profissionais da saúde sobre a prática de atividade física. IN: Congresso Brasileiro de Medicina de Família e Comunidade. 12:1339. Belém, 2013 Maio. Anais.

BERNARDO, A. F. B. et al. Caracterização das categorias de atividade física e associação com variáveis antropométricas de funcionários universitários. Revista brasileira Ciência e Movimento, Brasília, 21(2): 89-97, jun, 2013.

BIELEMANNL, R. M. et al. Consumo de alimentos ultraprocessados e impacto na dieta de adultos jovens. Revista de Saúde Pública, 49 (28): 1- 10, 2015.

CAVALCANTE, C. A. A. et al. Riscos ocupacionais do trabalho em enfermagem: uma análise contextual. Revista
Ciência, Cuidado e Saúde, Maringá, 5(1): 88-97, Jan/abr, 2006.

CONCEIÇÃO, T. V. et al. Valores de Pressão Arterial e suas Associações com Fatores de Risco Cardiovasculares em Servidores da Universidade de Brasília. Arquivos Brasileiros de Cardiologia, Brasília, 86(1): 26- 31, Jan. 2006.

COSTA, A. F. et al. A contribuição de um programa de ginástica laboral para a aderência ao exercício físico fora da jornada de trabalho. (s/d). Disponível em $<\mathrm{http}$ :// www.castelobranco.br/sistema/novoenfoque/files/07/08. pdf>, acesso em 06 de junho de 2013.

ELIAS, M. A.; NAVARRO, V. L. A relação entre o trabalho, a saúde e as condições de vida: negatividade e positividade no trabalho das profissionais de enfermagem de um hospital escola. Revista Latino-Americana de Enfermagem, Ribeirão Preto 14(4): 1-9, Jul/Aug. 2006.

FARIA JÚNIOR, A. G. Educação física no mundo do trabalho: ginástica de pausa, em busca de uma metodologia. In: MINISTÉRIO DA EDUCAÇÃO. Secretaria de Educação Física e Desportos, esporte e lazer na empresa/ Secretaria de Educação Física e Desporto. Brasília: MEC/ SEED, 1990. 221p.

FREIRE, C. B. et al. Qualidade de vida e atividade física em profissionais de terapia intensiva do sub médio São Francisco. Revista Brasileira de Enfermagem, 68(1):2631 , jan/fev 2015.

FRONZA, F. C. A. O; TEIXEIRA, L. R. Perfil dos profissionais da saúde que trabalham em hospitais: relação entre sintomas musculoesqueléticos e qualidade de vida. Revista Brasileira de Ciências da Saúde: (8) 24,53-61, abr/jun 2010 .

JESUS, G. M.; JESUS, E. F. A. Nível de atividade física e barreiras percebidas para a prática de atividades física entre policiais militares. Revista Brasileira de Ciências do Esporte, 34 (2): 433- 448, 2012.

KAC, G.; VELÁSQUEZ-MELÉNDEZ, G. A transição nutricional e a epidemiologia da obesidade na América Latina. Caderno de Saúde Pública, Rio de Janeiro, 19 (Sup. 1):S4-S5, 2003.

KARINO, M. E. et al. Cargas de trabalho e desgastes dos trabalhadores de enfermagem de um hospital-escola. Ciência, Cuidado e Saúde, 14(2):1011-1018, abr/jun, 2015.

LARA, M. A; SOARES, F. A; JUVÊNCIO, J. F. Qualidade de vida no trabalho - o papel da ginástica laboral. In: IV Workshop de análise ergonômica do trabalho - UFV; I Encontro mineiro de estudos em ergonomia. Viçosa, Minas Gerais, Junho de 2009.Anais.

LIMA JÚNIOR, J.; ALCHIERI, J. C.; MAIA, E. M. C.; 
Avaliação das condições de trabalho em hospitais de Natal, Rio Grande do Norte, Brasil. Revista Escolar de Enfermagem USP, 43(3): 670-676, 2009.

MALTA, D. C. et al. A construção da vigilância e prevenção das doenças crônicas não transmissíveis no contexto do Sistema Único de Saúde. Epidemiologia e Serviços de Saúde, Brasília, 15(1) : 47 - 65, 2006.

MANTOVANI, E. P. Atividade Física, Saúde e Envelhecimento. 65 -72, s/d. Disponível em $<$ http://www. fef.unicamp.br/fef/qvaf/livros/alimen_saudavel_ql_af/ diagnostico_funcamp/funcamp_cap9.pdf $>$, acesso em $15 \mathrm{de}$ maio de 2013.

MARIATH, A. B. et al. Obesidade e fatores de risco para o desenvolvimento de doenças crônicas não transmissíveis entre usuários de unidade de alimentação e nutrição.

Caderno de Saúde Pública, Rio de Janeiro, 23(4):897-905, abr, 2007.

MATOS, C. H.; PROENÇA, R. P. C. Condições de trabalho e estado nutricional de operadores do setor de alimentação coletiva: um estudo de caso. Revista de Nutrição, 16( 4): 493-502, 2003.

MORENO, C. R. C; FISCHER, F. M.; ROTENBERG, L. A saúde do trabalhador na sociedade 24 horas. Revista São Paulo em perspectiva, 17(1): 34- 43, 2003.

NETA, E. S. A. R.; FERNANDES FILHO, J.; CORTEZ, A. C. L. Nível de atividade física e estado nutricional de policiais militares na cidade de Floriao- PI. Revista Kinesis, 34 (1): 84-101, 2016.

PAFARO, R. C; MARTINO, M. M. F.; Estudo do estresse do enfermeiro com dupla jornada de trabalho em um hospital de oncologia pediátrica de Campinas. Revista da Escola de Enfermagem da Usp, São Paulo, 38(2):152-160, 2004.

RAMOS, C. V.; DUMITH, S. C.; CÉSAR, J. A. Prevalence and factors associated with stunting and excess weight in children aged 0-5 years from the Brazilian semi-arid region. Jornal de Pediatria, 91 (2): 175- 182, 2015.

REZENDE, F. A. C. et al. Indice de massa corporal e circunferência abdominal: associação com fatores de risco cardiovascular. Arquivos Brasileiros de Cardiologia, 87 (6): 728- 734, 2006.

ROCHA-BRISCHILIARI, S. C. et al. Doenças Crônicas não Transmissíveis e Associação com Fatores de Risco. Revista Brasileira de Cardiologia, 27(1):531-38, jan/fev, 2014.

SANTANA, L. L. et al. Cargas e desgastes de trabalho vivenciados entre trabalhadores de saúde em um hospital de ensino. Revista Gaúcha de Enfermagem, 34(1):64-70, 2013.
SANTICS, F. et al. Atividade física corporativa: colaborações para a saúde do trabalhador. Revista Digital, Buenos Aires, 13(125), Out. 2008.

SANTOS, I. S; DUARTE, E. C. Fatores de risco e proteção para doenças crônicas não transmissíveis na população adulta brasileira. Revista de Saúde Pública; 43(2): 5-6, 2009.

SILVA, B. M. et al. Jornada de trabalho: fator que interfere na qualidade da assistência de enfermagem. Revista Contexto Enfermagem, Florianópolis, 15(3): 442-448, jun/ set, 2006.

SILVA, R. S. et al. Atividade física e qualidade de vida. Ciência \& Saúde Coletiva, 15(1): 115-120, 2010.

SILVA, A. P.; QUEIROZ, E. S. O estresse e sua relação com a jornada de trabalho da enfermagem em unidade hospitalar. Periódico Científico do Núcleo de Biociências, 01(01): 33-50, ago.2011.

SILVA, A. A; ROTENBERG, L.; FISCHER, F. M. Jornadas de trabalho na enfermagem: entre necessidades individuais e condições de trabalho. Revista Saúde Pública, 45(6):11171126, 2011.

SILVA, J. V. F. et al. A relação entre o envelhecimento populacional e as doenças crônicas não transmissíveis: sério desafio de saúde pública. Cadernos de Graduação Ciências Biológicas e da Saúde, 2 (3): 91-100, Mai 2015.

SILVEIRA, N. R.; GATTELLI, P. J.; MOURA, C. B. Avaliação da percepção de risco frente a acidentes com perfurocortantes entre acadêmicos de enfermagem da universidade estadual do oeste do paraná. In: VII SEPECEL - Seminário de Ensino, Pesquisa e Extensão do Centro de Educação e Letras. Universidade Estadual do Oeste do Paraná, Campus Foz do Iguaçu, PR. Anais... Foz do Iguaçu, 2012.

SIQUEIRA, F. V. et al. Aconselhamento para a prática de atividade física como estratégia de educação à saúde. Caderno de Saúde Pública, Rio de Janeiro, 25(1):203-213, jan, 2009.

THOMAS, J. R.; NELSON, J. K.; SILVERMAN, S. J. Métodos de Pesquisa em Atividade Física. Ed.5.Porto Alegre: Artmed, 2007. 